\title{
„Selektive“ Fortpflanzung durch pränatale Diagnostik?
}

\author{
Christoph Rehmann-Sutter
}

Eingegangen: 25. Januar 2021 / Angenommen: 22. Juli 2021 / Online publiziert: 29. September 2021

(C) Der/die Autor(en) 2021

Zusammenfassung Die breite Einführung nicht-invasiver pränataler Tests (NIPT) sowie die Ausweitung der Testziele über Trisomien hinaus machen es notwendig, Sinn und Ziel der pränatalen Diagnostik (PND) als emergente soziale Praxis grundsätzlich zu diskutieren. Wenn, wie angenommen wird, PND nicht zu eugenischen Zwecken, sondern zur Stärkung der Autonomie dienen soll, muss gefragt werden, welche Bedeutung die Entscheidungen haben, ein bestimmtes zukünftiges Kind (nicht) zu gebären. Stephen Wilkinson hat vorgeschlagen, PND als eine Form ,selektiver Reproduktion“ zu verstehen. In diesem Paper wird geprüft, ob die Charakterisierung der Entscheidung nach PND als „Selektion“ zutrifft und welche moralischen Vorannahmen ihr zugrunde liegen.

Es zeigt sich, dass das Konzept der „,selektiven Reproduktion“ die Handlungen der PND inakkurat repräsentiert. Es beinhaltet zudem sowohl eine Abstrahierung als auch eine Distanzierung. Es nimmt an, dass Frauen und Paare entweder falls nötig mehrere Schwangerschaften planen, um ein gesundes Kind zu erzeugen, oder sich als Ausführende einer selektiven Strategie auf der Populationsebene verstehen. Die Einschränkung der ethischen Diskussion auf das Problem der Selektion verdeckt zwei wichtige Problemfelder, die die konflikthaltige Situation der PND aus der Perspektive der Frau oder des Paares charakterisieren: die Schwangerschaft als persönliche Beziehung und den Akt des Abbruchs der Schwangerschaft. Aufgrund seiner impliziten Normativität wird ,,selektive Fortpflanzung“ als sinnvolle Bezeichnung für die Praxis der PND zurückgewiesen.

Dieser Beitrag bezieht sich auf eine vorgängig publizierte Kurzfassung: Rehmann-Sutter C (2021) Should prenatal screening be seen as 'selective reproduction'? Four reasons to reframe the ethical debate. J Perinat Med. https://doi.org/10.1515/jpm-2021-0239, publiziert unter CC BY 4.0 Lizenz (https://creativecommons.org/licenses/by/4.0/).

Prof. Dr. phil. dipl. biol. Christoph Rehmann-Sutter $(\bowtie)$

Institut für Medizingeschichte und Wissenschaftsforschung, Universität zu Lübeck,

Königstraße 42, 23552 Lübeck, Deutschland

E-Mail: christoph.rehmannsutter@uni-luebeck.de 
Schlüsselwörter Pränatale Diagnostik · Nicht-invasive pränatale Tests (NIPT) • Selektive Reproduktion · Selektion · Humangenetik · Soziale Praktiken · Stephen Wilkinson

\title{
"Selective" reproduction through prenatal diagnosis?
}

\begin{abstract}
Objective The broad introduction of noninvasive prenatal tests (NIPT) in recent years as well as its potential to include testing targets beyond trisomies ask for a reflection about the purpose of prenatal diagnosis (PND) as an emergent social practice. One prominent proposal in bioethical literature is to subsume PND to "selective" reproductive practices. In this paper, the accuracy and the moral presuppositions of this characterization are examined.

Method Moral analysis of the argumentative strategy in Stephen Wilkinson's seminal publications on "selective reproduction"; explication of the assumptions connected to the characterization of prenatal diagnosis as a selective act; discussion of PND with social practice theory.

Results "Selection" misrepresents the decision after prenatal diagnosis. It also involves a problematic abstraction and distancing: it contains the assumption that the women and the couple either plan potentially several pregnancies from which they would choose one to bring to term, or that the women and the couple should understand themselves as executives of a selective strategy on the population level. By restricting the bioethical discussion on the issue of selection it ignores two relevant fields of moral concern that characterize moral perception in the situation of prenatal diagnosis: the ongoing pregnancy as a personal relationship and the ending of fetal life.

Conclusion On the grounds of this implicit normativity, selective reproduction is rejected as a meaningful description of the purposes of prenatal diagnosis.
\end{abstract}

Keywords Prenatal diagnosis - Noninvasive prenatal tests (NIPT) - Selective reproduction $\cdot$ Selection $\cdot$ Human genetics $\cdot$ Social practices $\cdot$ Stephen Wilkinson

\section{Einleitung}

Die breite Einführung nicht-invasiver pränataler Tests (NIPT) seit 2013 vor allem für Trisomien 13, 18 und 21 sowie für einige andere relativ häufige genetische Krankheiten, sowie die Ausweitung möglicher Testziele durch DNA-Sequenzierung hat, wie es Munthe (2015) ausgedrückt hat, zu einer ,,neuen Landschaft“ der Pränataldiagnostik (PND) geführt. In dieser Situation ist es notwendig geworden, über Sinn und Ziel der pränatalen Diagnostik als eine emergente soziale Praxis der Reproduktion grundsätzlich zu diskutieren (de Jong und de Wert 2015; Hashiloni-Dolev et al. 2019). Mit der weltweiten Verbreitung pränataldiagnostischer Möglichkeiten seit den 1970er-Jahren, um Dispositionen und Eigenschaften eines Ungeborenen zu erkennen, sind Mutterschaft, Elternschaft und die Familien vor veränderte Voraussetzungen gestellt: Die Geburt ist auch mit Entscheidungen darüber verbunden, 
welche Kinder zur Welt kommen sollen. Für diejenigen, die pränatale Diagnostik in Anspruch nehmen, ist die Fortpflanzung an Entscheidungen auf der Grundlage von Untersuchungsergebnissen über den Embryo oder Fötus gebunden. Diejenigen, die auf Tests verzichten, nehmen das Nichtwissen bewusst in Kauf.

Die geschichtlich immer noch junge Praxis der PND hat nicht nur die Voraussetzungen für neuartige autonome Entscheidungen geschaffen, sondern auch moralische und weltanschauliche Unsicherheiten ausgelöst. Diese verlangen nach kommunikativer und moralischer Vergewisserung. Was bedeutet es, was man in der Fortpflanzung mit PND tut? Eine Frage ist in der Diskussion schon früh aufgetaucht (Parens und Asch 1996; Shakespeare 1998; Haker 2002; vgl. historisch Löwy 2017): Ist die PND eine Form von ungerechter „Selektion“, eine im besten Fall freiwillige Form von „Eugenik“ (,liberale Eugenik“; Habermas 2001) oder kann sie auch im Sinn einer neu ausgelegten Verantwortung für das Wohl der geborenen Kinder verwendet werden? Kann sie die Welt sogar gerechter machen, wie Buchanan, Brock, Daniels und Wikler argumentierten (Buchanan et al. 2000)?

Seit den 1980er-Jahren haben die Methoden der humangenetisch unterstützten Fortpflanzung in unterschiedlichen Gesellschaften und Kulturen zunehmend auch die Aufmerksamkeit der Sozialwissenschaften auf sich gezogen (Rothman 1986; Gregg 1995; Rapp 1999; Samerski 2002; Meskus 2012; Lou et al. 2018). Die beiden Sozialanthropolog:innen Tine Gammelthoft und Ayo Wahlberg haben vorgeschlagen, die fortpflanzungsmedizinischen Verfahren PND, Amniozentese, Chorionzottenbiopsie, die nicht-invasiven genetischen Tests, aber auch die diagnostischen Ultraschalluntersuchungen, die Auswahl von Samen- und Eizellen und die genetischen Carriertests als ,selektive reproduktive Technologien“ zusammenzufassen, um sie hinsichtlich ihrer Regulierung, ihrer lokalen Bedeutungen, und den sozialen Praktiken in verschiedenen Gesellschaften vergleichend untersuchen zu können. Ihnen allen ist nämlich gemeinsam, dass sie es, formal betrachtet, Eltern möglich machen zu bestimmen, welche Kinder sie bekommen möchten: ,to choose the children they want to enter this world“ (Gammelthoft und Wahlberg 2014, S. 202; Wahlberg und Gammelthoft 2018). Es ist diesen Autor:innen nicht entgangen, dass darin eine Provokation steckt, die zu Untersuchungen Anlass geben soll. Die „,neue Landschaft“ der PND besteht, wie man heute gut begründet behaupten kann (Munthe 2015) vor allem darin, dass durch Screenings und immer neue, verbesserte Testangebote Informationsgrundlagen für individualisierte Entscheidungen geschaffen werden, die mehr und mehr auf den Schultern der Schwangeren und Ihrer Partner liegen. Wenn dem so ist, so gibt es starke Gründe, die Frage zu stellen, was diese Verantwortung beinhaltet, und was die Entscheidungen bedeuten, die individuell verantwortet werden müssen. Ist der Sinn dieser Entscheidungen tatsächlich die Selektion unter möglichen Nachkommen? ${ }^{1}$ Was würde mit diesem Begriff der „Selektion“ dann aber impliziert?

Schon vor dem Aufkommen der Amniozentese haben Menschen versucht, den Ausgang ihrer Fortpflanzung aktiv zu beeinflussen. Eine Auswahl, sofern überhaupt ausgewählt wurde, konnte aber erst nach der Geburt stattfinden. Infantizid, Im-Stich-

\footnotetext{
${ }^{1}$ Birnbacher (2006, S. 317) sieht in der Pränataldiagnostik mit Schwangerschaftsabbruch „eindeutig ein[en] Fall von Selektion“. Ranisch (2017) folgt diesem Wortgebrauch.
} 
Lassen und selektives Vernachlässigen von Kindern aufgrund von Merkmalen, die sich am geborenen Kind zeigen, waren seit der Antike in verschiedenen Gesellschaften verbreitet (historische Nachweise bei Langer 1974), wurden aber sowohl im Judentum, Christentum als auch im Islam vehement abgelehnt (Giladi 1990). Das Aufkommen und die weltweite Verbreitung ,selektiver reproduktiver Technologien“" verschob die Entscheidung, ob ein bestimmtes zukünftiges Kind leben soll, vor die Geburt zurück und konnte so wesentlich breitere Akzeptanz gewinnen. In einem Kontext liberaler Abtreibungsregelungen scheint ein Verbot der PND ebenso wenig begründbar wie eine moralische und rechtliche Verpflichtung, ein Kind trotz festgestellter schwerer Beeinträchtigungen auszutragen (Löwy 2017). ${ }^{2}$

Wenn der Begriff der ,selektiven reproduktiven Technologien“ als deskriptive Kategorie verwendet wird, muss damit keine Wertung verbunden sein. Wenn versucht wird, den Schwangerschaftsabbruch nach einer PND als ,selektiver Abbruch“ von „elektiven Abbrüchen“ zu unterscheiden, die unabhängig von den spezifischen Eigenschaften des Ungeborenen sind und allein darin begründet sind, dass die Schwangerschaft ungewollt ist (Bleisch und Büchler 2020, S. 145; so auch im TAB-Bericht zu NIPT: Kollek und Sauter 2019), ist kein anderes Interesse im Spiel als zwei Rechtskategorien klar zu unterscheiden, also in Deutschland Abbrüche nach § 218a, Ziff. 1 oder 2. ${ }^{3}$ Anders ist es aber, wenn der Begriff der Selektion in der Bioethik zur Erklärung der sozialen und moralischen Bedeutung von fortpflanzungsmedizinischen Praktiken verwendet werden soll. Dieser Verwendung des Selektionsbegriffs wende ich mich nun zu.

Ausgehend von einer Reihe von Arbeiten vor allem von Stephen Wilkinson (Wilkinson 2010; Wilkinson und Garrard 2013; Tarkian 2020) sind in den letzten Jahren Fragen der moralischen Legitimität auch pränataldiagnostischer Tests im Rahmen einer ,selektiven“ Praxis diskutiert worden. Diese Autor:innen behaupten, dass die Pränataldiagnostik offensichtlich eine Variante von „selektiver Reproduktion“ darstelle (,,an instance of selective reproduction“; Wilkinson 2010, S. 3). Damit findet, wie ich zeigen möchte, eine materiell fragwürdige, nicht selbstverständliche Sinnzuschreibung der sozialen Praxis der PND statt, die voraussetzungsreich und ethisch problematisch ist. Sie soll deshalb zur Diskussion gestellt werden. Ich werde dazu die Pränataldiagnostik (im Sinn von Theodore Schatzki 2001) als eine soziale Praxis thematisieren, die aus Tun und Sagen, aus Technik und Sprache besteht, also auch die Bezeichnungen als Sinnauslegungen mit umfasst. Damit möchte ich auf einen blinden Fleck in einigen Ethikansätzen zur Pränataldiagnostik hinweisen: die implizite Normativität der Beschreibung der pränataldiagnostischen Praxis als eine „,selektive Fortpflanzung“.

\footnotetext{
2 Eine Pflicht zum Austragen fehlt in den meisten Ländern. Eine Ausnahme ist Polen nach der Entscheidung des Verfassungsgerichts vom 22. Oktober 2020, welche den Schwangerschaftsabbruch auch auf Grund von schweren Anomalien für verfassungswidrig erklärte.

3 Der Begriff der „,selektiven Abtreibung“ stammt ursprünglich aus einem anderen Kontext. Er wurde seit den 1980er-Jahren vor allem für die „Reduktion“ von Mehrlingsschwangerschaften verwendet, die bei gesundheitlichen Risiken für die Frau, bei pränatal erkannten Schädigungen eines der Mehrlinge oder zur Erhöhung der Überlebenschance des oder der verbleibenden Föten als indiziert gilt (Eberbach 1989, S. 270).
} 
Diese Diskussion ist deshalb wichtig, weil der Sinn der sozialen Praxis (wie auch die regulierenden Normen) gesellschaftlich getragen und vermittelt wird und sich in den persönlichen Entscheidungen der Einzelnen wiederfindet. Es kommt deshalb in der gesellschaftlichen Wirklichkeit der Humangenetik und Fortpflanzungsmedizin darauf an, wie man sagt, ,was man da tut“, d.h. in welche Rahmung das diagnostische Handeln gestellt wird: weil der Name dem Tun einen bestimmten Sinn verleiht, der moralische Bedeutungen enthält.

\section{„Selektion“ in der bioethischen Diskussion}

Simon Reader (2017, S. 25) ordnet Wilkinsons Position der ,orthodox liberal eugenic view of selective reproduction in philosophical bioethics“ zu. Der Grund, weshalb ich Wilkinson für die vorliegende Analyse als zentralen Autor gewählt habe, ist der, dass sich in Wilkinsons Arbeiten besonders klar und scharf eine typische Argumentationslogik findet, die direkt mit der Charakterisierung der PND als Praxis der „Auswahl“ zusammenhängt. Das von Wilkinson vorgelegte Argumentationsschema ist zu einer der zentralen Referenzen in der englischsprachigen liberalen Ethik der Humangenetik geworden (s. Prusak 2013; Bayefsky und Jennings 2015; Kaposy 2018; Cavaliere 2020; kritisch Teubl 2011), wird auch im deutschsprachigen Raum rezipiert (Tarkian 2020; Ranisch 2017; Henning 2020) und bietet sich für eine genauere kritische Untersuchung an, weil es besonders geeignet ist, um die moralischen Implikationen der normativen Vorstellung einer selektiven Fortpflanzung freizulegen.

Wie Hannes Foth in seiner Studie zur Bedeutung der NS-Vergangenheit im biopolitischen Diskurs in Deutschland aufgezeigt hat, wurde der Begriff der Selektion in der Diskussion um NIPT meistens in einer negativen Bedeutung verwendet: um zu sagen, was mit einer routinisierten, breiten Anwendung von NIPT zu befürchten ist. Der Begriff wurde selten als Beschreibung für die gegenwärtig akzeptierte Praxis der PND verwendet, sondern zur Kennzeichnung einer problematischen Entwicklung: Es gehe darum zu vermeiden, dass mit NIPT eine selektive, eugenische Praxis entsteht. „Avoiding selection and eugenics should be regarded as a central part of the German post-war moral identity“ (Foth 2021, S. 8).

Wilkinson bezeichnet nun die PND affirmativ als „,selektives“ Vorgehen. Er bestimmt die PND normativ als selektive Reproduktion und analysiert sie gemeinsam mit anderen Formen der selektiven Reproduktion (wie die PID oder die Entscheidung, lieber später als jetzt ein Kind zu zeugen) hinsichtlich ihrer ethischen Vertretbarkeit. Tatjana Tarkian (2020) rekonstruierte dieses Argument mit dem entsprechenden deutschen Wort „Auswahl“ (vgl. kritisch: Beck (2020), MitscherlichSchönherr (2020) und Rothhaar (2020)) und gibt folgende Definition der ,Auswahl zukünftiger Kinder":

„Mit der Auswahl zukünftiger Kinder ist die Entscheidung für ein (mögliches) zukünftiges Kind zuungunsten eines anderen (möglichen) zukünftigen Kindes gemeint - also der Versuch, ein (mögliches) zukünftiges Kind durch ein anderes (mögliches) zukünftiges Kind zu ,ersetzen“"“(Tarkian 2020, S. 112).

Das entspricht Wilkinsons Definition: 
„By ,selective reproduction“ I mean the attempt to create one possible future child rather than a different possible future child.“ (Wilkinson 2010, S. 2).

In diese Kategorie fällt insbesondere die PND und der ,selektive Schwangerschaftsabbruch" mit oder ohne NIPT, wie auch die Präimplantationsdiagnostik (PID), aber auch wenn , jemand ein Kind mit Partner A und nicht mit B [zeugt], oder [...] mit Partner A hier und heute und nicht in drei Monaten“ (Tarkian 2020, S. 113). In allen diesen Fällen hängt es von einer Entscheidung ab, welches mögliche Kind in der Zukunft existieren wird. Tim Henning (2020) wies zu Recht auf die problematische Gleichsetzung der Entscheidung über die Existenz „,bloß möglicher“ Personen mit Entscheidungen über die Existenz derjenigen Individuen, ,die aus diesem oder diesem oder ... Embryo werden“ hin, die mit der Subsumption in der allgemeinen Rubrik der ,selektiven Reproduktion“"verbunden ist. Sein Argument ist, dass der Fötus, der zu einer Person werden kann, über deren zukünftige Existenz entschieden wird, bereits jetzt aktual existiert, während nicht gezeugte Kinder bloß möglich sind (in diesem Sinn auch Mitscherlich-Schönherr 2020). ${ }^{4}$

\section{Sinn der PND als soziale Praxis}

Eine „soziale Praxis“ wird (Schatzki 1996, 2001; Reckwitz 2002) als eine Verbindung typischer Handlungsverläufe und geteilter sprachlicher Sinnzuschreibungen verstanden: ,a temporally unfolding and spatially dispersed nexus of doings and sayings“ (Schatzki 1996, S. 86). Die verwendeten Begriffe, Vergleiche, Metaphern und Narrative (,sayings“) sind für das sinnhafte Verständnis der im Rahmen einer Praxis getroffenen Entscheidungen und der vollzogenen Handlungen (,doings“) maßgeblich. Weil die Beschreibungen einer Praxis handlungsleitend sind, sind nicht nur die einzelnen Entscheidungen ethisch reflexionsbedürftig, sondern auch die in einer Gesellschaft eingerichteten Routinen und Üblichkeiten samt ihren Symbolisierungen.

Zwischen der allgemein normativen Ebene der gesetzlichen Regelungen und der Ebene individueller Entscheidungen über die Verwendung von PND liegt, wie die Theorie sozialer Praxis zeigt, vermittelnd eine Ebene geteilter Verständnisse, sozialer Erwartungen und Vorstellungen davon, was in einer Situation typischerweise richtig ist zu tun. Die Ethik kann sich deshalb nicht darauf beschränken, bloß zu klären, was erlaubt ist und welche Verbote sich rechtfertigen lassen. Auch diese Begriffe und sinngebenden Narrative auf der mittleren Ebene müssen kritisch betrachtet werden, weil sie eine Praxis beschreiben, leiten, begleiten und sprachlich repräsentieren. Oft enthalten sie implizite Verweise auf Normen und Werte. PND muss als eine im Medium der Sprache sozial vermittelte Praxis angesehen werden.

Indem sie im Einzelnen selbstbestimmt vollzogen wird und sich durch einzelne Zusätze und technische Innovationen in einer Entwicklung befindet, wird die PND als soziale Praxis nicht von Einzelnen jeweils in einer Situation neu erfunden. Es ist eine mehr oder weniger vorgesehene, mit Hilfe von Dispositiven in der Gesellschaft eingerichtete Art und Weise, Fortpflanzung zu tun. Die Leute wissen: Heute macht

\footnotetext{
4 Vgl. zur Selektion im Interesse des Kindeswohls den Beitrag von Ruth Denkhaus in diesem Heft.
} 
„man“ es so. Einzelne können sich auf dieses Gemeinsame stützen und sich in ihm wiederfinden. In Situationen der Ungewissheit und des Orientierungsbedarfs können sie sich auf etwas stützen, was in der Gesellschaft üblich und weitgehend anerkannt ist. Sie sehen, was andere tun und hören, weshalb sie es für sinnvoll halten.

Weil soziale Praktiken aus Tun und Sprechen bestehen, sind typische Beschreibungen einer sozialen Praxis nicht nur ein Name für eine Praxis (und als solche weniger wichtig als ihr Inhalt), sondern sie bilden selbst Teil dieser Praxis. Wie in einem anderen Bereich der Medizin die typische Beschreibung „,gutes Sterben“ eine normativ gehaltvolle Semantik aufweist, die der „kommunikativen Vergewisserung einer sonst vergleichsweise ungewissen Praxis“ dient (Breitsameter 2020, S. 346), weist eine typische Beschreibung der PND als „selektive Reproduktion“ eine normativ gehaltvolle Semantik auf, die der kommunikativen Vergewisserung einer konflikthaften und angefochtenen, deshalb moralisch ungewissen Praxis dient.

Welche Bedeutung hat eine Handlung? Diese Frage stellt sich nicht nur für die Sozialwissenschaftler:innen und Ethiker:innen, sondern für jede handelnde Person selbst. Mit Charles Taylor gehe ich davon aus, dass Menschen sich selbst in Situationen und im sozialen Kontext interpretierende Wesen sind (,we have to think of man as a self-interpreting animal“; Taylor 1971, S. 16). Menschen wollen - und müssen - mit ihren Handlungen jeweils einen Sinn verbinden. Der Sinn einer Handlung ist nicht nur in der Zweckrationalität zu suchen, sondern sie beinhaltet jeweils eine Interpretation der Situation, aus der heraus die Handlung erwächst, in Bezug auf die eine Zielsetzung und die Wahl der Mittel adäquat sein kann.

Für die Handelnden, wie Taylor ausführt, kann die Situation auch verwirrend erscheinen und Widersprüche enthalten. Eine Handlung kann aus einem adäquaten Verständnis der Widersprüche heraus erfolgen, in denen die Akteure den Sinn ihrer Situation sehen (Taylor 1971, S. 14). Die kommunikative Vergewisserung über den Sinn des diagnostischen Handelns beeinflusst, wie Menschen die diagnostischen Techniken in ihrem Lebenskontext verstehen, welche Auslegungsangebote sie im Rahmen der persönlichen Entscheidungen akzeptieren und welche sie zurückweisen. „Selektive Reproduktion“ kann als ein solches Auslegungsangebot verstanden werden und ist im Rahmen der emergenten sozialen Praxis der PND ethisch zu thematisieren. Sie stellt gleichzeitig eine normativ gehaltvolle Auslegung dessen dar, was wir, wie es Hille Haker formulierte, ,mit einer ,verantwortlichen Elternschaft“ unter den Bedingungen der PND überhaupt meinen“" (Haker 2012, S. 35).

\section{Die Einklammerung des Privaten im Namen der Autonomie}

\section{Struktur und Stärke von Wilkinsons Argument}

Unmittelbar auf die allgemeine Definition von „selektiver Reproduktion“ als Auswahl möglicher zukünftiger Kinder folgt bei Wilkinson der Verweis auf die subjektiven Gründe, weshalb Frauen oder Paare ein mögliches zukünftiges Kind einem anderen vorziehen und sie deswegen selektive Reproduktion praktizieren: The reason ,is normally that one possible future child is, in some way, more desirable than the alternatives“ (Wilkinson 2010, S. 2). Wünschbarkeit könne unterschiedliche Mo- 
tive enthalten und die Frage, was als wünschbar gelten soll, sei kontrovers. Aber es gebe ein Motiv, das relativ unkontrovers sei, nämlich die Auswahl, um Krankheiten zu vermeiden. Wenn ein mögliches zukünftiges Kind eine schwere, lebensverkürzende Krankheit haben wird, während ein anderes sie nicht haben wird, sei es die vernünftigste Option, dafür zu sorgen, dass das krankheitsfreie Kind erzeugt wird:

,ensuring that the disease-free child is created seems like the sensible thing to do (all other things being equal)." (Wilkinson 2010, S. 3).

Es gebe „low tech“ selektive Fortpflanzung, z.B. wenn eine Frau einen Partner oder einen Samenspender wählt, oder wenn sie über den Zeitpunkt ihrer Fortpflanzung entscheidet, sowie ,high tech“ Möglichkeiten, zu denen die PID und die PND gehören. Das Prinzip der Auswahl bleibe jedoch dasselbe.

Im Rahmen eines liberalen Moral- und Staatsverständnisses muss nun nicht die Freiheit, etwas zu tun, begründet werden, sondern eine Einschränkung dieser Freiheit. Wilkinson nennt das die Prämisse der Permissibilität (Presumption of Permissibility). Zusammen mit einer zweiten Annahme, nämlich die eines „formalen“ politischen Liberalismus (Schematic Political Liberalism), die auf Mill zurückgeht, führt dies zu dem von Wilkinson gewählten Vorgehen: Die Untersuchung beginnt mit der Hypothese, dass selektive Reproduktion vertretbar ist (permissible). Sodann müssen die stärksten Einwände gegen die Freiheit zur selektiven Reproduktion identifiziert und der Reihe nach geprüft werden. Wenn sie sich alle als nicht stichhaltig genug erweisen, um die selektive Reproduktion (oder Teile davon) für unvertretbar zu halten, ist das Ergebnis, dass sie erlaubt sein soll.

Wilkinson gibt explizit an, welches die Gründe sind, die eine Handlung seiner Ansicht nach unvertretbar machen. Mill selbst erkannte bekanntlich bloß den Schaden für andere („Harm Principle“) als Kriterium an, um Eingriffe des Staates in die privaten Freiheiten zu rechtfertigen. Wilkinson geht über Mill hinaus und hält auch Handlungen für unvertretbar, die zwar andere nicht schädigen, sie aber ausbeuten oder instrumentalisieren: ,harmless, but nontheless wrongful, exploitation or instrumentalization“ (Wilkinson 2010, S. 11).

Im Text identifiziert er eine Reihe von Gründen, die eine Einschränkung möglicherweise erlauben könnten. Die wichtigsten sind Folgende (vgl. auch Wilkinson und Garrard 2013): (1) die Pflicht zur bedingungslosen Liebe als parentale Tugend; (2) das Wohl zukünftiger Kinder; (3) die gleiche Berücksichtigung von Menschen mit Krankheiten und Behinderungen; (4) das Eugenik-Argument, (5) mögliche schädliche Konsequenzen für Menschen mit Behinderungen und (6) das expressive Argument, d.h. die Sorge, dass eine selektive Reproduktionspraxis eine Botschaft sendet, welche die Rechte von Menschen mit Behinderungen tangiert oder sich sonst ungünstig auf sie auswirkt. Nach Wilkinson sind diese Gründe wie folgt zu bewerten:

(1) Eine Pflicht zur bedingungslosen Liebe könne sich höchsten auf existierende Kinder erstrecken, nicht aber auf prospektive Kinder. Ähnlich auch Tarkian: Die Frage ist die, ,ob Schwangere verpflichtet sind, eine Auswahl gesunder zukünftiger Kinder zu unterlassen, oder ob Paare schlechte zukünftige Eltern wären, wenn sie sich diagnostischer Methoden bedienen möchten“ (Tarkian 2020, S. 118). Dies kann mit dem Ideal der bedingungslosen Hinwendung aber nicht begründet werden. 
(2) Das Wohl zukünftiger Kinder stellt in der Tat eine Motivation für die selektive Reproduktion dar, insofern nämlich Krankheiten oder Behinderungen vermieden werden, die dem Wohl abträglich wären. Eine Auswahl, die dem Wohl der Ausgewählten entgegenläuft, könnte sich nicht rechtfertigen; daraus entsteht aber kein grundsätzlicher Einwand gegen die selektive Reproduktion.

(3) Die gleiche Berücksichtigung von Menschen mit Krankheiten und Behinderung wird durch die selektive Fortpflanzung nicht angetastet. Dies wäre nur dann der Fall, wenn sie mit einer Diskriminierung oder Schlechterbehandlung von Geborenen einhergeht. Föten fallen nicht unter das Gleichstellungsgebot. Sofern eine Diskriminierung Geborener verhindert wird, entsteht kein Grund zur Einschränkung der selektiven Fortpflanzung.

(4) Das Eugenik-Argument lässt Wilkinson deshalb nicht gelten, weil nicht jede Form von Eugenik (Wilkinson definiert sie als Verbesserung des menschlichen Genpools; vgl. aber Rubeis 2018) unmoralisch sei. Solange funktionale Beeinträchtigungen vermieden werden und die Person, die Beeinträchtigungen hat, nicht abgewertet wird, und wenn die Autonomie der Frauen gewahrt bleibt, könne die Sorge, dass die breite Praktizierung von selektiven Methoden in einem technischen Sinn „eugenisch“ sei, keinen hinreichenden Grund bilden, diese Praxis zu verbieten.

(5) Im Argument, dass die breite Anwendung selektiver Methoden schädliche Konsequenzen für Menschen mit Behinderungen haben kann, sei ein Kern Wahrheit enthalten. Wenn es nämlich weniger Menschen mit einer bestimmten Form von Behinderung gibt, werden vielleicht in der Gesellschaft weniger Ressourcen für sie bereitgestellt. Aber gegenüber dem Anliegen der Vermeidung von Leiden durch Prävention der Geburt von Kindern mit Behinderungen sei auch dieses Bedenken $\mathrm{zu}$ vage, um ein Verbot zu begründen.

(6) Das expressive Argument schließlich sei nur wirksam für eine enge Gruppe von Fällen, in denen die Motivation der Eltern die ist, dass sie durch ihren Akt, oder durch die Auslegung, die sie ihrem Akt geben, den Wert der Existenz von Menschen mit Behinderungen in Frage stellen. Dies sei aber nicht bei allen Eltern der Fall, die selektive Reproduktion praktizieren.

Das Ergebnis der Prüfung ist laut Wilkinson negativ. Es gibt keine hinreichend gewichtigen moralischen Gründe, um die der ,selektiven Reproduktion“ zugerechneten Handlungen allgemein als moralisch unzulässig zu bezeichnen. Entsprechend ist ein gesetzliches Verbot nicht gerechtfertigt.

Schwachpunkte dieser Argumentation zeigen sich allerdings, sobald man sie über die Gesetzesdiskussion über alle Formen der ,selektiven Reproduktion“ hinaus ausweitet und spezifischer auf die Praxis der PND, also auf eine der Varianten selektiver Reproduktion hin konkretisiert. Wenn ich das im Folgenden tue, sehe ich gleichwohl keinen Grund, die Konklusion von Wilkinson, Garrard und Tarkian anzugreifen, dass innerhalb eines liberalen Staatsverständnisses, das aus guten Gründen den Schwangerschaftsabbruch mit sozialer Indikation zulässt, keine hinreichend starken ethischen Gründe für ein Verbot der PND zu sehen sind.

Dass mit dem Aufweis der moralischen Zulässigkeit der PND in der Ethik nicht alles getan ist, ist auch in liberaler Sicht unumstritten. Es stellen sich eine Reihe von begleitenden Aufgaben - für die Behörden und für die Akteure im Gesundheitswesen. Oft wird darauf hingewiesen, dass die wünschenswerte Autonomie und eine 
wirklich freie und informierte Entscheidung ein anspruchsvolles und nicht einfach zu realisierendes Konzept ist (de Jong und de Wert 2015). Johnston und Zacharias (2017) sprechen von einer ,reproductive autonomy worth having“, um sie von anderen, weniger erstrebenswerten, formalen Formen von Autonomie zu unterscheiden, die sie als ,get out of my way approach“ charakterisieren. Es muss für die Frau Handlungssicherheit entstehen. D.h. es muss konkret vermieden werden, dass Entscheidungen über Tests und Schwangerschaftsabbruch überstürzt, unter Druck oder auf der Grundlage mangelhafter Informationen zustande kommen. Die Auswirkungen der Aufnahme von NIPT in die Mutterschaftsrichtlinien und der Kostenübernahme durch die Kassen sind auch in dieser Hinsicht im Rahmen der resultierenden Praxis sorgfältig und wachsam zu begleiten (Rehmann-Sutter und Schües 2020). Mit Vardit Ravitsky kann außerdem dafür argumentiert werden, dass durch die Etablierung der PND, um Frauen durch ein vorhersehbar schlechtes Leben der Kinder mit Behinderungen nicht ungebührend unter Druck zu setzen, für die Gesellschaft eine Pflicht erwächst, nämlich die Pflicht zur bedürfnisgerechten Unterstützung und gesellschaftlichen Inklusion derjenigen Kinder, die genau mit denjenigen Konditionen geboren werden, gegen die Tests verbreitet werden (Ravitsky 2017; RehmannSutter 2020; ähnlich auch Tarkian 2020, S. 121).

\section{Multiple Akteurperspektiven}

Die Akteurperspektiven sind wesentlich komplexer als es in der Diskussion um ein Verbot aufscheint; persönliche Entscheidungen können nicht nur von allgemeinen moralischen Forderungen bestimmt werden, die wir gegenseitig an alle richten, sondern auch von persönlichen, situativen moralischen Erwägungen (vgl. García et al. 2012; Meskus 2012). Die Ethik hat es also nicht nur mit der Regulierungsperspektive zu tun. Es geht auch um ein Verständnis der Situationen, die mit PND geschaffen werden - in ihren multiplen Akteurperspektiven. Die ethisch relevanten Gesichtspunkte in der Perspektive der Schwangeren und der Paare, die NIPT wie andere Verfahren der PND benutzen oder ablehnen, sind mit der Frage des Schwangerschaftsabbruchs verbunden und nicht damit zu erledigen, dass es dafür in der Gesellschaft eine liberale Regelung geben soll. Es geht um ihre eigenen Entscheidungen. Schwangere Frauen, die einen Test machen lassen, wissen, dass der Test zu einem Befund führen kann, der zu einer Entscheidung über die Weiterführung der Schwangerschaft führt. Ärzt:innen und genetische Berater:innen wissen, dass dies die Frauen wissen. Auch wenn die Frauen und Paare, die PND im Hinblick auf einen möglichen Abbruch in Anspruch nehmen, den Schwangerschaftsabbruch für moralisch zulässig halten und viele deshalb ein moralisches Recht auf Schwangerschaftsabbruch verteidigen würden, bedeutet das nicht, dass sie ihre persönliche Entscheidung zu einem Abbruch für moralisch belanglos halten.

Wilkinsons Argumentationsschema, das sich auf die Frage der Zulässigkeit (,permissibility") beschränkt, berücksichtigt von drei moralischen Problembereichen (1. Wahl zwischen zukünftigen Kindern zur Vermeidung einer schweren Behinderung oder Krankheit, 2. Abbruch der in der Schwangerschaft wachsenden, persönlichen Mutter-Kind-Beziehung, 3. Tötung des Fötus) nur den einen, nämlich die Wahl zwischen zukünftigen Kindern. Sie lässt die anderen beiden Bereiche 
moralischer Sorge der Frauen und Paare außer Acht. Die Argumentationsweise, die Wilkinson skizziert, ist aus diesem Grund reduktionistisch.

Dies zeigt sich auch in Interviews mit Betroffenen. Stina Lou et al. fanden in einer qualitativen Studie mit 21 Paaren in Dänemark, die sich nach einem pränataldiagnostischen Befund von Trisomie 21 für einen Abbruch ihrer Schwangerschaft entschieden hatten, dass alle Paare die existenzielle Last dieser Entscheidung in der einen oder anderen Weise spürten. Die meisten zeigten ein detailliertes Faktenwissen über das Down Syndrom und die Diversität der DS-Phänotypen und waren sich bewusst, dass sie sich gegen ein möglicherweise gutes Leben ihres Kindes entschieden hatten. „Secondly, all couples addressed the decision as burdensome due to its irreversibility and potential cause of future regret." Dennoch bereute aber keines der Paare die Entscheidung zum Abbruch der Schwangerschaft im Moment des Interviews. „Thirdly, for all couples, the termination of pregnancy implied not only the loss of a wanted child, but also of a desired future. All expressed a sense of arbitrariness and grief that the desired future had been shattered and had to be re-pursued“" (Lou et al. 2018, S. 1233f.). Die Interviewten beschäftigten sich also intensiv mit den Fragen des Abbruchs ihrer Schwangerschaft und des Neinsagens zum Leben des Fötus. Wenn es in der Ethik darum gehen soll, die ethischen Implikationen und die moralischen Bedeutungen der PND zu klären, muss sie auch die wichtigsten Fragen diskutieren, die sich in der Sicht der betroffenen Paare stellen.

\section{Wahl und Auswahl}

Teil von Wilkinsons Argumentationsstrategie ist, die Handlung der PND zusammen mit anderen Handlungstypen (low und high tech) unter einem Überbegriff der selektiven Reproduktion zu subsumieren. Einzelne der subsumierten Beispielhandlungen haben zu öffentlichen Kontroversen geführt (PID, Genome Editing ...), während andere kaum je unter dem Gesichtspunkt der Selektivität diskutiert wurden (Partnerwahl, Zeitpunkt des Kinderhabens). Man könnte darin eine Absicht vermuten. Wilkinson könnte die kontroversen Handlungsarten als ähnlich wie andere, kaum je moralisch hinterfragte Handlungen erscheinen lassen wollen. Moralisch signifikante Unterschiede würden damit eingeebnet.

Ich möchte hier aber nicht diesen Punkt stark machen, sondern auf einen anderen, aus meiner Sicht problematischeren Punkt hinweisen, der sich durch die Subsumption in die allgemeine Handlungskategorie der selektiven Reproduktion ergibt: die von Wilkinson vorgenommene moralische Parallelsetzung von PND mit PID. Die beiden Techniken unterscheiden sich darin, welche Art von Entscheidung oder Wahl sie beinhalten. Während PID klar eine Auswahl ist, nämlich eine Selektion zwischen mehreren vorliegenden Embryonen, ist die PND mit einer Entscheidung über das Austragen einer Schwangerschaft verknüpft. Dieser kann eine nächste Schwangerschaft folgen, muss aber nicht. Bei PID hingegen wird zu einem Zeitpunkt aus mehreren Individuen ausgewählt. Bei PND kann höchstens eine serielle Entscheidung erfolgen, die zu einer Auswahl führt (s. Abb. 1). Dieser Unterschied ist ethisch relevant, wenn er die moralische Bedeutung der Entscheidung betrifft. Diese muss aufgewiesen werden. 
Abb. 1 a Auswahl (von Embryonen) bei PID; b serielle Entscheidung (über Föten) bei PND

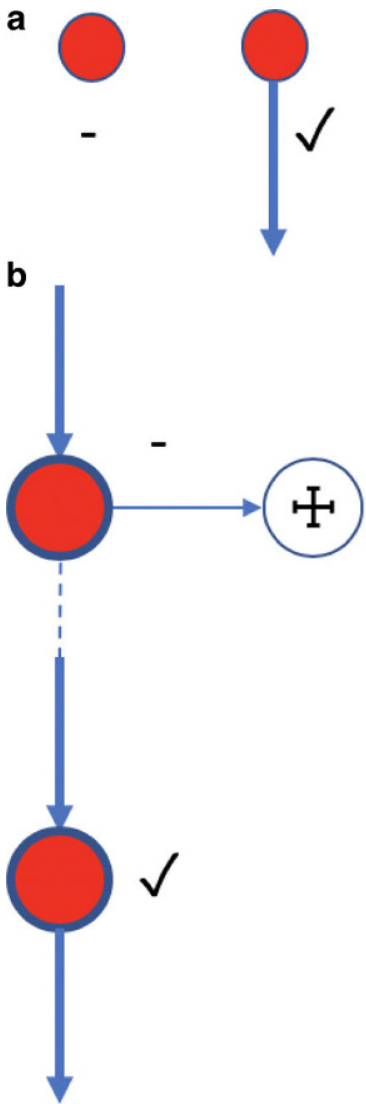

Bevor wir dies untersuchen, ist aber schon fraglich, ob der Begriff der Selektion oder Auswahl überhaupt auf den Abbruch einer Schwangerschaft nach PND korrekt angewendet werden kann. Selektion stammt von lat. seligere $=$ auslesen. Das setzt mehrere Optionen oder Entitäten voraus, mindestens zwei, unter denen ausgewählt wird. ${ }^{5}$ Das Wort kann demnach nicht die Entscheidung über eine einzige Strategie oder Entität bezeichnen.

Man könnte versuchen, die Anwendung des Begriffs Selektion für die PND mit drei Argumenten zu verteidigen:

(i) Nach PND besteht die Wahl zwischen „Abbruch oder nicht“, insofern gibt es tatsächlich zwei Optionen, zwischen denen eine gewählt wird. Dies ist aber nicht Selektion im Sinn von Wilkinson, der „,selektive Reproduktion“ als Auswahl zwischen möglichen zukünftigen Kindern definiert (s. oben).

(ii) Wenn PND einen schwerwiegenden Befund erbringt, könnte die Entscheidung über die Nichtfortsetzung der Schwangerschaft als eine „Abwahl“ dieses zukünftigen Kindes aufgefasst werden. ${ }^{6}$ Die Wahl eines Amtsträgers kann aber nur dann eine

\footnotetext{
5 Birnbacher (2006, S. 316): „welches von mehreren“.

6 Ich danke Christina Schües für diesen Hinweis.
} 
Wahl sein - und die Entscheidung gegen ihn eine Abwahl, wenn es eine Alternative gibt. Insofern ist auch die Abwahl bei fehlender Alternative keine echte Wahl, sondern präziser eine personbezogene Entscheidung gegen diesen Amtsträger - analog zur Entscheidung gegen dieses zukünftige Kind.

(iii) Tarkian (2020, S. 112) und Birnbacher (2006, S. 317) sprechen bei PND von einem „Ersetzen“ des Kranken durch ein gesundes Kind (Anführungszeichen auch im Original). Dies wäre tatsächlich eine sequenzielle oder diachrone Auswahl, setzt aber zwei Dinge voraus: (a) dass es nicht Platz für beide Kinder gibt, (b) dass eine nächste Schwangerschaft bereits in Aussicht steht, durch die ein gesundes Kind geboren werden kann. Wenn diese Bedingungen nicht erfüllt sind, ist es im Moment der Entscheidung aus der Perspektive der Frau oder des Paares im eigentlichen Sinn kein Ersetzen. Ein Ersetzen des kranken zukünftigen Kindes durch ein Gesundes könnte als eine zeitlich ausgedehnte Variante von Selektion angesehen werden, wenn aus zwei nacheinander folgenden zukünftigen Kindern eines ausgewählt wird. Diese Annahme ist aber fragwürdig: (i) Sie ist abhängig von der Entscheidung der Frau oder des Paares, dass es so sein soll; es ergibt sich nicht aus der Natur der Sache selbst. (ii) Es ist unsicher und wird auch nicht in jedem Fall gewünscht. Es ist daher fraglich, ob die serielle Entscheidung zutreffend eine „Auswahl“/,Selektion“ genannt werden kann. - Eher ist zu fragen, unter welchen Voraussetzungen eine solche Bezeichnung tatsächlich zutreffen würde. Erst eine bereits für die Eventualität geplante Sequenz von Schwangerschaften, bei denen jeweils PND angewendet wird und zu einer Auswahl von Geburten, zu einem „Ersetzen“ dieses zukünftigen Kindes durch ein nächstes führt, könnte Selektion genannt werden.

Der Selektionsbegriff könnte zudem auch eine populationsgenetische Sicht insinuieren. Man verlässt dann aber die Perspektive der Schwangeren und würde behaupten: Die verbreitete Anwendung der PND in der Bevölkerung führt im Effekt zu einer Selektion zwischen Schwangerschaften, die ausgetragen werden, und solchen, die abgebrochen werden, also zu einer Selektion der geborenen Kinder. Wenn die einzelne Frau oder das einzelne Paar diese Bezeichnung verwenden, würden sie sich virtuell in die Position von ausführenden Organen einer populationsgenetischen Planung versetzen.

In beiden Perspektiven - eine Sequenz von Schwangerschaften oder der Blick auf die Bevölkerung - geht es nicht um eine Entscheidung über ,diese“ Schwangerschaft. Aus diesem Grund ist die Rede von „Selektion“, ,selektiver Reproduktion“, „Auswahl“" oder von einem „selektiven Schwangerschaftsabbruch“ nach PND begrifflich inkorrekt.

Zusammenfassend: Die Nivellierung des Unterschiedes zwischen der Auswahl unter mehreren Entitäten zu einem Zeitpunkt (bei der PID) oder der seriellen Wahl oder der Entscheidung über ein zukünftiges Kind (bei der PND), die mit der Subsumption unter die Handlungskategorie der selektiven Reproduktion vorausgesetzt ist, impliziert, dass die konkrete Entscheidung über den einzelnen Fötus und die einzelne Schwangerschaft nicht so sehr zählen soll wie die Auswahl, die aber im Fall der PND eine übergeordnete abstrakte Perspektive über mehrere Schwangerschaften hinweg oder die Populationsperspektive beinhaltet. Darin besteht die implizite Normativität des Selektionsbegriffs, wenn er für die PND verwendet wird. Diese Hierarchisierung ist aus der Perspektive des moralischen Gewissens der Frau oder 
des Paares, die ja die Entscheidung autonom treffen sollen, eine ausgesprochen problematische Gedankenanordnung: Es gibt eine Beziehung zu einem Fötus als ein aktuell werdendes Wesen, das sich zur Geburt hin entwickelt. Diese Beziehung wird zugunsten einer abstrakten Situationsdefinition in den Hintergrund gedrängt.

\section{Selektion vor der Geburt?}

Die Frage hat sich umgedreht: Wann wird die PND zu einer Praxis, die man im Sinn von „selektiver Reproduktion“ als Selektion vor der Geburt bezeichnen kann, oder sogar muss? Aus der Perspektive der Frau oder des Paares betrachtet, ist nach der dargelegten Begriffsklärung davon auszugehen, dass die PND in den meisten Fällen nicht als Selektion bezeichnet werden kann. Wilkinson nimmt die Situation der PID als Modell, wo tatsächlich - im hellen Licht unter dem Binokular, unterstützt von Ergebnissen der Untersuchung von entnommenen Zellen der Embryos zwischen mehreren embryonalen Wesen eines oder zwei ausgewählt werden. Ob PID aber als Modell zur Auslegung der Situation der PND geeignet ist, in der eine Schwangerschaft besteht, über die entschieden wird, bleibt fraglich.

In Bezug auf das einzelne Wesen (Fötus, werdendes Kind ...) führt der Befund aus einer PND die Schwangere zu einer Entscheidung, die Schwangerschaft abzubrechen oder weiterzuführen - aus Gründen, die dieses zukünftige Kind, sie selbst und ihre Familie betreffen. Es ist eine Entscheidung, die aus der Beziehung erwächst, welche diese Schwangerschaft darstellt. Olivia Mitscherlich-Schönherr (2020, S. 142) nennt eine solche Entscheidung deshalb eine „genuine Beziehungsentscheidung“. Christina Schües (2020, 2016) erklärt die Schwangerschaft und die Geburt bereits vor dem Ereignis der Entbindung als ein Beziehungsgeschehen. In der Entscheidung aus dieser Beziehung kann die Hoffnung auf eine nächste Schwangerschaft mit einem gesunden Kind durchaus mitschwingen. Um diese Entscheidung aber als eine Selektionsentscheidung erscheinen zu lassen, müssten beide Schwangerschaften, die bestehende und die zukünftige, gleich bewertet werden. Dabei werden zwei gedankliche Bewegungen ausgeführt:

(i) Die Geburt wird zu einem „Projekt“ gemacht, das mehrere Schwangerschaften beinhalten kann, die schon geplant sind, um bestimmte Tests zu durchlaufen. Mitscherlich-Schönherr (2020, S. 139) zeigte, dass darin kulturell bedingte Vorannahmen stecken: ein ,subjektivistisch verkürztes Verständnis der Geburt als ein ,Projekt“ der Reproduktion, das in einer ,Kette von Entscheidungen “ umgesetzt wird“.

(ii) Eine Distanzierung vom aktuellen Schwangersein und von der Erwartung dieses Kindes. Das Schwangersein wird verstanden als ein Versuch, ein Kind ohne feststellbare Krankheiten und Behinderungen zu erzeugen. Er kann auf Anhieb gelingen; er kann aber auch scheitern. Die personale Beziehung zum werdenden Kind wird aufgeschoben bis nach Abschluss der Testreihen die Entscheidung über die Fortführung der Schwangerschaft fest steht. Die aktuelle Schwangerschaft besteht erst mal auf Probe (,tentative pregnancy“; Rothman 1986).

Dass einigen Autor:innen, Wilkinson folgend, den Selektionsbegriff heute bewusst zur moralischen Bestimmung der PND mit einem eventuell folgenden Abbruch der Schwangerschaft nach medizinischer Indikation einsetzen, beinhaltet deshalb genau besehen eine normative Umdeutung der Fortpflanzung als Praxis. Das 
muss nicht immer bewusst geschehen; einige mögen den Begriff bloß dafür verwenden, um damit die moralische Schärfe dieser Diagnostik zum Ausdruck zu bringen, die zu Abbruchsentscheidungen führen kann. ${ }^{7}$

Zumindest im deutschsprachigen Raum ist es fast nicht möglich, den Begriff der Selektion von der Erinnerung an die Greuel des NS-Regimes zu lösen. ${ }^{8}$ Der Begriff kann auch eine implizite Referenz auf sozialdarwinistisches Gedankengut enthalten (,survival of the fittest“). Entsprechend wird der Begriff auch meistens im negativen Sinn zur Kritik der Ausweitung der PND, ihrer Routinisierung oder der Finanzierung aus den Krankenkassen verwendet. In kritischer Bedeutung kommt er auch in englischsprachiger Literatur vor, wenn etwa die Historikerin der PND Ilana Löwy (2017, S. 187) beschreibt, wie PND im späteren 20. Jahrhundert in vielen industrialisierten Ländern zu einem technologischen Imperativ und auch zum Versorgungsstandard wurde, begleitet von ethischen Kontroversen, ,frequently centered on the risks of a gradual and imperceptive sliding into increasingly selective reproduction." Die selektive Reproduktion ist bei Löwy das Resultat eines zunehmenden Abgleitens. So ist auch die Wortverwendung bei Zhang (2020).

PND als selektive Praxis zu deuten, verleiht der Praxis der Fortpflanzung mit PND deshalb eine bestimmte Bedeutung, die sich nicht aus der Natur der Sache ergibt und die moralisch gehaltvoll ist: Es wird ein Fötus erzeugt, getestet und sodann über das weitere Austragen oder den Abbruch entschieden, mit der Aussicht, einen nächsten zu erzeugen, der den ersten ersetzen wird. Oder Frauen und Paare verstehen sich in ihrer Schwangerschaft als ausführende Organe einer populationsbezogen epidemiologischen Strategie. Diese Deutung des Sinnes der sozialen Praxis der PND als serielle Erzeugung auf Vorbehalt oder als Teil eines bevölkerungsmedizinischen Projekts, schließt andere Deutungen aus, die primär am Konflikt in der einzelnen Schwangerschaftsbeziehung orientiert sind, oder relativiert sie. Dies widerspricht einer starken rechtsethischen Tradition in Deutschland, wie z.B. in der dominanten Auslegung der PID in der Zeit vor dem Urteil des BGH von 2010: Die PID sollte vor allem deshalb verboten sein, weil sie eine selektive Praxis darstelle. Ein Beleg dafür ist ein Text des Medizinrechtlers Adolf Laufs von 1998 (Laufs 1998, S. 1753): „Die Produktion eines Embryos unter dem Vorbehalt der Tötung bei Qualitätsmängeln und die eugenische Selektion verbieten sich.“ Die negative und die positive (,eugenische“) Selektion seien vor dem Hintergrund der deutschen Rechtskultur ausgeschlossen. Dies müsste analog auch für die PND gelten.

Wenn man diesen Überlegungen folgt, wird deutlich, dass mit der selbstverständlich werdenden Rede von selektiver Fortpflanzung als Bezeichnung für die soziale Praxis der PND eine ethisch problematische historische Kontinuität zu einer früher praktizierten Form von Eugenik impliziert wird. Als (dünnes - vgl. Thomas und Rothman 2016) Abgrenzungskriterium bleibt nur die Abwesenheit von staatlichen Vorschriften.

\footnotetext{
7 Ich danke Claudia Schumann für diesen Hinweis.

8 „Nach der Selektion gingen zwanzigtausend in die Gaskammern“ (Zywulska 1988, S. 323). Zur Bedeutung der Erinnerung an den Nationalsozialismus in der gegenwärtigen Bioethik vgl. Foth (2021).
} 


\section{Ergebnis}

Wilkinsons Argumentation führt zum Resultat, dass PND und andere Varianten der selektiven Reproduktion grundsätzlich zulässig sein sollen. Sein Begründungsvorschlag nimmt aber, wie ich gezeigt habe, eine sowohl sachlich inkorrekte als auch ethisch problematische Repräsentation der Praxis der PND als „Selektion“ in Kauf. Die Subsumption der PND in die Handlungskategorie der ,selektiven Reproduktion" ist einerseits deskriptiv fehlerhaft, weil sie die vorgeburtliche Untersuchung und die Entscheidung über die Fortsetzung einer Schwangerschaft als Auswahl zwischen mehreren zukünftigen Kindern oder als Ausübung einer populationsgenetischen Strategie darstellt und damit der Frau oder dem Paar ein selektives Motiv unterstellt. Eine Auswahl unter mehreren zukünftigen Kindern geschieht bei der PID oder bei der Auswahl von Samenspendern. Die Verwendung des Begriffs auch für PND impliziert eine Veränderung im Selbstverständnis der Schwangeren und der zukünftigen Eltern, sowie eine problematische Festlegung der Bedeutung der PND als soziale Praxis.

Die Behandlung der PND als ,selektive Reproduktion“ führt andererseits zur Ausblendung einer Reihe wichtiger moralischer Fragen aus der ethischen Diskussion, nämlich derjenigen Fragen, die sich nicht im Rahmen der Klärung stellen, welche Freiheitseinschränkungen in einem liberalen Staat vertretbar sind. Diese Fragen stellen sich aus multiplen Akteurperspektiven: der Frau, des Paares und der beteiligten Fachpersonen. Die Suche nach alternativen begrifflichen Rahmungen, die für diese Perspektiven Raum bieten, müsste von der Beziehung ausgehen, welche die Schwangerschaft auszeichnet, und die Entscheidung über die Fortsetzung oder den Abbruch der Schwangerschaft als Fürsorgekonflikt in der nicht ersetzbaren Beziehung dieser Schwangerschaft interpretieren.

Mein Anliegen und mein Argument waren deshalb nicht nur begrifflicher Art. Es geht um die Gestaltung der Interdependenz von Tun und Sagen, von Handlung und Sprache in einer sozialen Praxis. Die Normativität der Konzepte steht zur Debatte, die zur Repräsentation der PND Verbreitung finden. „Selektive Reproduktion“ ist als Charakterisierung (und damit als Teil) der sozialen Praxis der PND nicht nur deshalb problematisch, weil das Wort „Selektion“ entweder sozialdarwinistisch oder eugenisch und damit negativ konnotiert ist und schwierige historische und politische Bedeutungen mit sich trägt. Es trägt dazu bei, die PND als Teil einer ,liberalen Eugenik“ (Habermas 2001; Agar 1998) auf der Basis der Präferenzen der Eltern hinsichtlich der Wünschbarkeit von Eigenschaften mehrerer möglicher zukünftiger Kinder zu (re)konstruieren, die solange moralisch als vertretbar gilt, solange sie auf dem Prinzip der freien und autonomen Entscheidung der Einzelnen beruht.

Die Umdeutung der PND als ,,selektive Reproduktion“ ist außerdem auch deshalb problematisch, weil die Vokabel ,Selektion“ in diesem Kontext eine ablehnende Haltung gegenüber Menschen mit Behinderungen beinhaltet (disablism; Goodley 2014). Selektion ist der Vorgang eines Aus- und Einsortierens, nicht eine Entscheidung, die in einer Schwangerschaft getroffen wird. Das verstärkt die Sorge, die oft mit ,expressives Argument" oder ,disability rights critique“ bezeichnet wird (Heyer 2018; vgl. Rubeis und Steger 2019), und macht es schwieriger, diese Sorge mit dem Argument für unbegründet zu erklären, dass die Entscheidung gegen die Geburt eines 
Kindes mit Behinderung keine ablehnende Haltung gegenüber den existierenden Kindern mit Behinderung enthält. Wie auch Wilkinson und Garrard (2013, S. 16) anerkennen, ist die Haltung, die hinter der PND steht, wichtig um zu entscheiden, ob das expressive Argument Gehalt hat. Sie nennen als Beispiele für eine behindertenfeindliche Haltung Eltern, die lieber gar kein Kind als ein Kind mit einer Behinderung haben oder die glauben, dass die Welt besser wäre, wenn es keine Menschen mit Behinderung gibt. Dieses Argument der Haltung bezieht sich aber auch auf die Wahl der Semantik der selektiven Reproduktion.

Wenn man Wilkinsons Argumentationsvorschlag nur als Begründung der moralischen und rechtlichen ,permissibility“ ansieht, wenn er also bloß begründen soll, was per Gesetz verboten werden darf und was erlaubt sein sollte, kommt ihm einige Plausibilität zu. Ein liberaler Rechtsstaat sollte den medizinisch begründeten Schwangerschaftsabbruch wie auch einige andere selektive Praktiken nicht kriminalisieren. Das bedeutet aber nicht, dass man versucht hat, ernsthaft etwas von der ethischen Problematik aus der vielfältigen Perspektive der Frauen, der Paare und der Beziehungen zu verstehen. Bioethik hat nicht nur die Aufgabe, die Grenzen staatlicher Beschränkungen der privaten Autonomie zu klären, sondern ihr Diskurs soll auch Raum schaffen für die ethischen Überlegungen und Konflikte, die für die Frauen und Paare wichtig sind, die diese Entscheidungen nach genetischer Beratung treffen müssen, oder für die Medizinalpersonen, die sie dabei beraten und versorgen. Eine pauschale Klassifizierung ihrer Entscheidungen in die Kategorie der Selektion tut denjenigen Frauen und Paaren Unrecht, die ihre Erwägungen nicht am Bild der diachronen Auswahl orientieren und eine Schwangerschaft auf Probe eingehen, sondern nach einer heute oft aus der routinemäßigen Schwangerenbetreuung folgenden PND in einen moralischen Konflikt geraten und diesen aus der Beziehungssituation der Schwangerschaft heraus deuten.

Die liberalistische Vorstellung von der Gesellschaft als einer Assoziation freier Individuen, die durch Gesetze miteinander verbunden und durch sie eingeschränkt werden, ist unterkomplex, weil sie die Vergesellschaftung von Individuen in sozialen Praktiken und die relationale Konstitution ihres Selbst und ihrer Autonomie kaum berücksichtigt. Frauen, Paare und die Provider von pränataler Genetik orientieren sich an geteilten Repräsentationen der Tätigkeit der PND, deren Bedeutungen normativ gehaltvoll sind. Diese Bedeutungen müssen diskutiert werden als Sinnkonstrukte, die wir in der Gesellschaft miteinander teilen. Ethische Argumentationen, die sich auf die Semantik von Beschreibungen richten, prägen auch die Motive der Menschen, die an der Praxis teilnehmen und sich in ihr orientieren. Gleichzeitig sind Eckpunkte für eine Kritik der Formen der sozialen Praxis der PND (und allgemeiner der pränatalen Genetik) sichtbar geworden, die sich in der Zukunft durch eine Veränderung und Verstärkung von Routinen ergeben können: Die Praxis der PND ist problematisch und entsprechend ethisch zu kritisieren, wenn bzw. insofern sie eine selektive Reproduktion darstellt.

Danksagung Diese Arbeit wurde ermöglicht von der DFG (RE-2951/3-1: Meanings and Practices of Prenatal Genetics in Germany and Israel - PreGGI). Für Recherchen danke ich Marina Frisman, für Hinweise zu früheren Fassungen des Manuskripts Ruth Denkhaus, Julia Inthorn und den KollegInnen in der PreGGIStudiengruppe: Hannes Foth, Yael Hashiloni-Dolev, Tamar Nov Klaiman, Anika König, Aviad Raz, Stefan Reinsch, Christina Schües, wie auch der AG „Ethische Fragen am Lebensanfang“ der AEM. 
Funding Open Access funding enabled and organized by Projekt DEAL.

Open Access Dieser Artikel wird unter der Creative Commons Namensnennung 4.0 International Lizenz veröffentlicht, welche die Nutzung, Vervielfältigung, Bearbeitung, Verbreitung und Wiedergabe in jeglichem Medium und Format erlaubt, sofern Sie den/die ursprünglichen Autor(en) und die Quelle ordnungsgemäß nennen, einen Link zur Creative Commons Lizenz beifügen und angeben, ob Änderungen vorgenommen wurden.

Die in diesem Artikel enthaltenen Bilder und sonstiges Drittmaterial unterliegen ebenfalls der genannten Creative Commons Lizenz, sofern sich aus der Abbildungslegende nichts anderes ergibt. Sofern das betreffende Material nicht unter der genannten Creative Commons Lizenz steht und die betreffende Handlung nicht nach gesetzlichen Vorschriften erlaubt ist, ist für die oben aufgeführten Weiterverwendungen des Materials die Einwilligung des jeweiligen Rechteinhabers einzuholen.

Weitere Details zur Lizenz entnehmen Sie bitte der Lizenzinformation auf http://creativecommons.org/ licenses/by/4.0/deed.de.

\section{Einhaltung ethischer Richtlinien}

Interessenkonflikt C. Rehmann-Sutter gibt an, dass kein Interessenkonflikt besteht.

Ethische Standards Für diesen Beitrag wurden vom Autor keine Studien an Menschen oder Tieren durchgeführt. Für die aufgeführten Studien gelten die jeweils dort angegebenen ethischen Richtlinien.

\section{Literatur}

Agar N (1998) Liberal eugenics. Public Aff Q 12:137-156

Bayefsky M, Jennings B (2015) Regulating preimplantation genetic diagnosis in the United States. The limits of unlimited selection. Palgrave Macmillan, New York

Beck B (2020) Reproduktive Freiheit für wen? Z Ethik Moralphilosophie 3:127-135

Birnbacher D (2006) Selektion von Nachkommen. In: Birnbacher D. Bioethik zwischen Natur und Interesse. Suhrkamp, Frankfurt a. M., S 315-335

Bleisch B, Büchler A (2020) Kinder wollen. Über Autonomie und Verantwortung. Hanser, München

Breitsameter C (2020) Die Semantik des ,guten Sterbens“ aus ethischer Perspektive. Ethik Medizin $32: 331-350$

Buchanan A, Brock DW, Daniels N, Wikler D (2000) From chance to choice. Genetics and justice. Cambridge Univ. Pr., Cambridge

Cavaliere G (2020) The problem with reproductive freedom. Procreation beyond procreators' interests. Med Health Care and Philos 23(1):131-140

Eberbach WH (1989) Pränatale Diagnostik - Fetaltherapie - selektive Abtreibung: Angriffe auf $\S 218$ a Abs. 2 Nr. 1 StGB (embryopathische Indikation). Juristische Rundsch 1989(7):265-273. https:// doi.org/10.1515/juru.1989.1989.7.265

Foth H (2021) Avoiding "selection"? References to history in current German policy debates about noninvasive prenatal testing. Bioethics. https://doi.org/10.1111/bioe.12880

Gammelthoft TM, Wahlberg A (2014) Selective reproductive technologies. Ann Rev Anthropol 43:201-216

García E, Timmermans DRM, van Leeuwen E (2012) Parental duties and prenatal screening: does an offer of prenatal screening lead women to believe that they are morally compelled to test? Midwifery 28:e837-e843

Giladi A (1990) Some observations on infanticide in medieval Muslim society. Int J Middle East Stud 22(2): 185-200

Goodley D (2014) Dis/ability studies: theorising disablism and ableism. Routledge, London

Gregg R (1995) Pregnancy in a high-tech age. Paradoxes of choice. New York University Press, New York, London

Habermas J (2001) Die Zukunft der menschlichen Natur. Auf dem Weg zu einer liberalen Eugenik? Suhrkamp, Frankfurt am Main 
Haker H (2002) Ethik der genetischen Frühdiagnostik. Sozialethische Reflexionen im menschlichen Lebensanfang. Mentis, Paderborn

Haker H (2012) Verantwortliche Elternschaft und pränatale Diagnostik. In: Vorgeburtliche Untersuchungen. BZgA FORUM 2, S 32-36

Hashiloni-Dolev Y, Nov Klaiman T, Raz A (2019) Pandora's pregnancy: NIPT, CMA, and genome sequencing - A new era for prenatal genetic testing. Prenat Diagn 39(10):859-865. https://doi.org/10. $1002 /$ pd.5495

Henning T (2020) Selektive Reproduktion, ethischer Aktualismus und Moralität de re. zphf 67:5-34

Heyer K (2018) Prenatal testing and disability rights: challenging 'genetic genocide. Stud Law Polit Soc $76: 101-129$

Johnston J, Zacharias RL (2017) The future of reproductive autonomy. Hastings Cent Rep 47(S3):6-11

de Jong A, de Wert G (2015) Prenatal screening: an ethical agenda for the near future. Bioethics 29(1):46-55

Kaposy C (2018) Choosing Down syndrome: Ethics and new prenatal testing technologies. MIT Press, Cambridge

Kolleck A, Sauter A (2019) Aktueller Stand und Entwicklungen in der Pränataldiagnostik. Endbericht zum Monitoring. TAB-Arbeitsbericht Nr. 184. Büro für Technikfolgen-Abschätzung beim Deutschen Bundestag, Berlin

Langer W (1974) Infanticide: a historical survey. Hist Child Q 1(4):353-365

Laufs A (1998) Arzt und Recht - Fortschritte und Aufgaben. NJW 1998(24):1750-1761

Lou S, Carstensen K, Petersen OB, Palmhøj N, Hvidman L, Lanther MR, Vogel I (2018) Termination of pregnancy following a prenatal diagnosis of Down syndrome: a qualitative study of the decisionmaking process of pregnant couples. Acta Obstet Gynecol Scand 97:1228-1236

Löwy I (2017) Imperfect pregnancies. A history of birth defects \& prenatal diagnosis. Johns Hopkins Univ. Pr, Baltimore

Meskus M (2012) Personalized ethics: the emergence and the effects in prenatal testing. BioSocieties 7:373-392

Mitscherlich-Schönherr O (2020) In Verteidigung der Rückhaltlosigkeit der menschlichen Geburt. Z Ethik Moralphilosophie 3:137-150

Munthe C (2015) A new ethical landscape of prenatal testing: individualizing choice to serve autonomy and promote public health: a radical proposal. Bioethics 29(1):36-45

Parens E, Asch A (Hrsg) (1996) Prenatal testing and disability rights. Georgetown University Press, Washington DC

Prusak BG (2013) Parental obligations and bioethics: the duties of a creator. Routledge, New York

Ranisch R (2017) Kritik der liberalen Eugenik. Ethik und Ideengeschichte der selektiven Reproduktion. Diss. Universität Düsseldorf

Rapp R (1999) Testing women, testing the fetus: the social impact of amniocentesis in America. Routledge, New York

Ravitsky V (2017) The shifting landscape of prenatal testing: between reproductive autonomy and public health. Hastings Cent Rep 47(Suppl 3):S34-S40

Reader S (2017) The ethics of choosing children. Palgrave Macmillan, Cham

Reckwitz A (2002) Toward a theory of social practices. A development in culturalist theorizing. Eur J Soc Theory 5:243-263

Rehmann-Sutter C (2020) Zur ethischen Bedeutung der vorgeburtlichen Diagnostik. In: Anselm R, Mitscherlich-Schönherr O (Hrsg) Gelingende Geburt in der Diskussion. De Gruyter, Berlin, S 263-287

Rehmann-Sutter C (2021) Should prenatal screening be seen as 'selective reproduction'? Four reasons to reframe the ethical debate. J Perinat Med. https://doi.org/10.1515/jpm-2021-0239

Rehmann-Sutter C, Schües C (2020) Die NIPT-Entscheidung des G-BA. Eine ethische Analyse. Ethik Medizin 32:385-403. https://doi.org/10.1007/s00481-020-00592-0

Rothhaar M (2020) Bedingte Akzeptanz? Moralische und rechtsethische Dimensionen der Ablehnung künftiger Kinder. Z Ethik Moralphilosophie 3:151-153

Rothman BK (1986) The tentative pregnancy: how amniocentesis changes the experience of motherhood. Viking Penguin, New York

Rubeis G (2018) Das Konzept der Eugenik in der ethischen Debatte um nicht-invasive Pränatal-tests (NIPT). In: Steger F, Orzechowski M, Schochow M (Hrsg) Pränatalmedizin: ethische, juristische und gesellschaftliche Aspekte. Alber, Freiburg/München, S 100-125

Rubeis G, Steger F (2019) A burden from birth? Non-invasive prenatal testing and the stigmatization of people with disabilities. Bioethics 33(1):91-97 
Samerski S (2002) Die verrechnete Hoffnung. Von der selbstbestimmten Entscheidung durch genetische Beratung. Westfälisches Dampfboot, Münster

Schatzki T (1996) Social practices: a Wittgensteinian approach to human activity and the social. CUP, Cambridge

Schatzki T (2001) Practice mind-ed orders. In: Schatzki T, Knorr-Cetina K, Savigny E (Hrsg) The practice turn in contemporary theory. Routledge, London, S 50-63

Schües C (2016) Philosophie des Geborenseins, 2. Aufl. Alber, München

Schües C (2020) Das Versprechen der Geburt. In: Anselm R, Mitscherlich-Schönherr O (Hrsg) Gelingende Geburt in der Diskussion. De Gruyter, Berlin, S 143-165

Shakespeare T (1998) Choices and rights: Eugenics, genetics and disability equality. Disabil Soc 13:665-681

Tarkian T (2020) Die Auswahl zukünftiger Kinder. Z Ethik Moralphilosophie 3:109-125

Taylor C (1971) Interpretation and the sciences of man. Rev Metaphys 25(1):3-51

Teubl KB (2011) Stephen Wilkinson, choosing tomorrow's children: the ethics of selective reproduction. $\mathrm{J}$ Value Inquiry 45:351-357

Thomas GM, Rothman B (2016) Keeping the backdoor to eugenics ajar? Disability and the future of prenatal screening. AMA J Ethics 18:406-415

Wahlberg A, Gammeltoft TM (2018) Introduction: kinds of children. In: Wahlberg A, Gammeltoft TM (Hrsg) Selective reproduction in the $21^{\text {st }}$ century. Palgrave Macmillan/Springer, Cham, S 1-24

Wilkinson S (2010) Choosing tomorrow's children: The ethics of selective reproduction. Oxford University Press, Oxford

Wilkinson S, Garrard E (2013) Eugenics and the ethics of selective reproduction. Keele University, Keele Zhang S (2020) The last children of Down syndrome. Atlantic 326:42-55

Zywulska K (1988) „Tanz Mädchen...“ Vom Warschauer Getto nach Auschwitz. Ein Überlebensbericht. Dtv, München 